\title{
Inhaled steroids and mortality in COPD: bias from unaccounted immortal time
}

\author{
S. Suissa
}

Inhaled steroids and mortality in COPD: bias from unaccounted immortal time. S. Suissa. (C) ERS Journals Ltd 2004.

ABSTRACT: A recent observational study, which suggested that inhaled corticosteroids (ICS) with or without long-acting bronchodilators are effective at reducing allcause mortality in chronic obstructive pulmonary disease (COPD) patients, may be subject to immortal time bias.

This bias was assessed using a population-based cohort of 3,524 newly treated COPD patients from Saskatchewan, Canada, observed from 1990-1999. Regular users of bronchodilators or ICS were followed for 3 yrs, during which time 860 deaths occurred. Cox's proportional hazards model was used to compare the hierarchical intention-totreat approach employed in the recent study, a technique subject to bias from two sources of immortal time, with the conventional intention-to-treat approach and the according-to-treatment approach.

The adjusted rate ratio of death using the hierarchical intention-to-treat approach was $0.66(95 \%$ confidence interval (CI) $0.57-0.76)$ for ICS use relative to bronchodilator use, compared with $0.75(95 \%$ CI $0.62-0.90)$ with the conventional intention-to-treat approach. Conversely, the rate ratio was 0.94 (95\% CI 0.81-1.09) with the according-to-treatment approach, which accounts for both sources of immortal time.

In this study, regular inhaled corticosteroid use in chronic obstructive pulmonary disease was not found to reduce all-cause mortality. Suggestion of this benefit from a previous observational study is the result of bias from unaccounted immortal time in its cohort design and analysis.

Eur Respir J 2004; 23: 391-395.
Division of Clinical Epidemiology, Royal Victoria Hospital, McGill University Health Centre, and the Depts of Epidemiology and Biostatistics and of Medicine, McGill University, Montreal, Canada.

Correspondence: S. Suissa, Division of Clinical Epidemiology, Royal Victoria Hospital, 687 Pine Avenue West, Ross 4.29, Montreal, Québec, Canada H3A 1A1.

Fax: 15148431493

E-mail: samy.suissa@clinepi.mcgill.ca

Keywords: Biases, cohort studies, drug effectiveness, epidemiology, immortal time, pharmacoepidemiology

Received: June 32003

Accepted after revision: October 52003

This research was funded by grants from the Canadian Institutes of Health Research (CIHR) and Fonds de la recherche en Santé du Québec (FRSQ). The acquisition of the database was funded by grants from AstraZeneca, Boehringer Ingelheim and GlaxoSmithKline. The author is the recipient of a Distinguished Investigator award from the CIHR. This study is based on de-identified data provided by the Saskatchewan Dept of Health. The interpretation and conclusions contained herein do not necessarily represent those of the government of Saskatchewan or the Saskatchewan Dept of Health.
The use of inhaled corticosteroids (ICS) in the treatment of chronic obstructive pulmonary disease (COPD) is highly debated, with important divergence in the data from randomised trials and observational studies [1]. Recent randomised trials have found that ICS treatment confers little or no benefit on lung function [2-7]. Although not part of the primary objective, two of these studies found a reduction in exacerbations or healthcare resource use among patients with moderate [7] or severe COPD [6]. Conversely, four recent observational studies have suggested that these drugs are extremely effective at preventing COPD morbidity and mortality.

Three of the observational studies, using an identical design, evaluated ICS dispensed after hospital discharge for COPD and found them to be very effective at preventing COPD morbidity and mortality [8-10]. The cohort design employed by these studies defined time zero (date of hospital discharge) independently of ICS exposure and used an intention-to-treat analysis. However, the current author has shown that this approach can lead to an important bias resulting from the introduction of immortal time and its misclassification with respect to exposure [11]. The study was replicated, using a different cohort of COPD patients, and a rate ratio (RR) of 0.69 was found for ICS, subject to bias. The RR became 1.00 when these sources of bias were properly attended to [11].

The fourth observational study used a different cohort design that appears to have accounted for immortal time [12]. This cohort study found that regular ICS use, given to newly diagnosed COPD patients, not necessarily after COPD hospitalisation, reduced the rate of all-cause mortality by $38-52 \%$ [12]. Unlike the other observational studies, however, time zero for cohort entry was defined by ICS exposure and the exposure was defined hierarchically. As a result of these definitions, it can be hypothesised that this study, using the intention-to-treat approach, also generated immortal time that was unaccounted for and that may have biased the findings.

In the present paper, it is shown that the intention-to-treat method used by this recently published study, by its approach to cohort formation and hierarchical exposure definition, introduces a bias involving immortal time that leads to 
significant distortion of the results. The nature of the bias is described and illustrated, and its impact is assessed using data from another population-based cohort of patients with COPD.

\section{Methods}

\section{Source of data}

The source population of COPD patients was identified using the computerised databases of Saskatchewan Health (Saskatchewan, Canada). These databases were developed as a result of the universal health insurance programme provided to all residents of this Canadian province since 1975 [13]. The base cohort, which has been previously described in more detail, was formed from all subjects newly treated for COPD in this population $[11,14,15]$. They were identified by having been dispensed three or more prescriptions on at least two different dates for a bronchodilator (short-acting $\beta_{2}$-agonist (SABA), inhaled anticholinergic or theophylline) within a 1-yr period between January 1, 1990 and December 31, 1997. Cohort entry was the date of the third prescription, at which point subjects were $\geqslant 55$ yrs of age. Subjects with any prescriptions for bronchodilators or other asthma drugs, including ICS, nasal corticosteroids, cromones (cromoglycate and nedocromil) and ketotifen, during the 5-yr period prior to cohort entry, were excluded. The cohort members were followed until December 31, 1999.

\section{Study cohort}

The study cohort was defined by the start of regular treatment with ICS or bronchodilators. Unlike the previously published study, which specifically evaluated the effect of fluticasone proportionate with or without salmeterol relative to bronchodilators, this study will focus simply on the effect of all ICS in contrast to bronchodilators. This is necessary since the use of long-acting $\beta$-agonists was very infrequent among cohort members. Nevertheless, the bias principle that is being illustrated will in fact gain in clarity with a simple comparison between two groups, namely ICS and bronchodilators, and not four groups.

As defined in the previous published study, regular treatment was taken to be the first dispensing of three or more prescriptions of ICS (in this study beclomethasone, fluticasone or budesonide) or three or more prescriptions of a bronchodilator from any one of the following classes: inhaled SABA, inhaled anticholinergics, xanthines or combined bronchodilators within a 6 -month period. Long-acting $\beta$ agonist use was ignored as it was too infrequent among cohort members. All subjects were then assumed to remain regular users of either bronchodilators or ICS until the end of follow-up, namely until the date of death, the date of emigration from the province, the date of end of coverage of the health insurance plan or December 31, 1999, whichever occurred first.

\section{Outcome}

The outcome was the occurrence of death from any cause during the follow-up. In conformity with the previously published study design, all deaths that occurred within 6 months of cohort entry were excluded.

\section{Exposure}

The previously published study used a hierarchical intentionto-treat definition for regular ICS exposure. Using this approach, the ICS group was first identified by the regular use of ICS, irrespective of whether a subject was already a regular user of bronchodilators, the reference exposure. The reference group for this hierarchical approach was thus composed of regular users of bronchodilators who were not regular users of ICS at any time during follow-up. This hierarchical approach may be biased by its exclusion of the immortal time that occurs during exposure to regular use of bronchodilators preceding the start of regular ICS exposure (fig. 1). Indeed, subjects could not have died during this period since they had to survive to become regular users of ICS. In addition, subjects were likely to be regular users of bronchodilators during this immortal time period.

To assess this bias, two alternative approaches of classifying exposure were considered. First, a conventional intentionto-treat approach was employed, based on the very first regular exposure identified after diagnosis. Using this method, regular users of bronchodilators were classified into the reference group even if they subsequently started regular ICS use. As a convention, subjects who became regular users of both ICS and bronchodilators on the same day were classified into the ICS group. Using this intent-to-treat approach, subjects remain classified in their exposure group until the end of follow-up. Secondly, an according-to-treatment approach was used, based on the actual exposure. Here, regular users of bronchodilators are classified into the reference group prior to starting regular ICS exposure, but only until they start ICS exposure. At that point, their person-time is classified into the ICS group until the end of follow-up.

Another source of immortal time arises from the definition of time zero. The recently published study used the date of the first of the three exposure-defining prescriptions within a 6-month period. The time span between the first and the third prescription is also immortal, as the subject must survive from the first to the third prescription to satisfy the definition of regular use. To avoid this bias, time zero should be defined as the date of the third of the three prescriptions. This alternative definition was used in the following analyses.

\section{Data analysis}

First, person-time of follow-up was classified as exposed to either ICS or bronchodilators (reference group) for each of the three different exposure definitions. Rates of death were
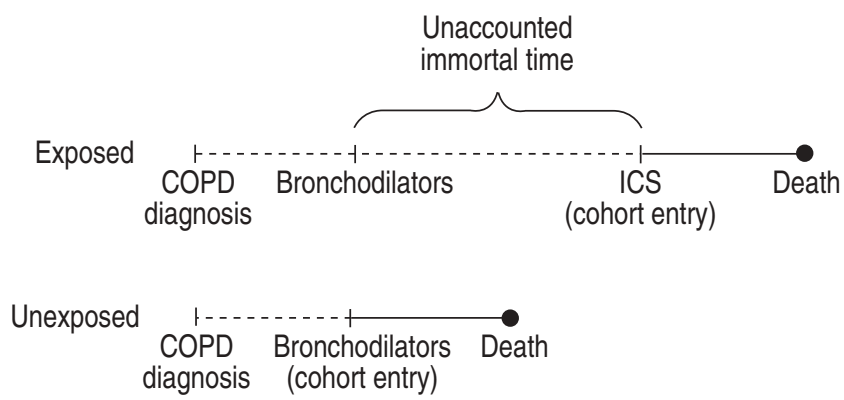

Fig. 1.-Order of events for typical exposed and unexposed subjects from the cohort who die during follow-up. The period of time from first regular bronchodilator use to first regular inhaled corticosteroid (ICS) use is immortal, since the subject must survive to receive ICS, but is unaccounted for by the design used in the previously published study [12]. COPD: chronic obstructive pulmonary disease. 
computed for each of the three approaches and crude RRs were estimated for regular ICS use relative to regular bronchodilator use. Cox's proportional hazards model with time-fixed and time-dependent exposure was used to crudely estimate these RRs, and they were adjusted for age at cohort entry and sex, as well as the occurrence of COPD hospitalisations and the use of other COPD medications dispensed during the 6-month period prior to cohort entry.

With respect to the definition of time zero, the time span between the first and third prescriptions defining time zero was first compared separately for bronchodilators and ICS, to verify if it differed for the two exposures. This alternative definition of time zero, namely the date of the third prescription instead of the first, was then used to estimate the crude and adjusted RRs for the three different approaches.

\section{Results}

The cohort included 5,645 subjects newly treated for COPD between 1990-1999, of which 3,682 subjects became regular users of bronchodilators or ICS at some point during followup. There were 1,738 subjects who were regular users of a bronchodilator and 951 regular users of ICS throughout the follow-up. There were also 993 subjects who were first regular users of a bronchodilator and in time became regular users of ICS. Table 1 describes these cohorts, after excluding all subjects who were followed-up for $<6$ months after cohort entry or who died within this period. The subjects who used ICS at any time were younger, more likely to have used SABA and to have been hospitalised for COPD prior to cohort entry.

Table 2 displays the crude rates of death estimated by the three different approaches. It should be noted that the crude RR of death for ICS exposure relative to bronchodilators goes from 0.53 for the hierarchical intention-to-treat approach, to 0.63 for the conventional intention-to-treat approach and to 0.69 for the according-to-treatment approach. The conventional intention-to-treat approach is based on a slightly larger number of subjects, since fewer subjects with $<6$ months of follow-up after cohort entry were excluded among those started on bronchodilators and followed by ICS. There were also fewer deaths because of the 3-yr follow-up limitation. Table 2 shows the impact of the immortal period of regular bronchodilator exposure occurring prior to the start of regular ICS use. This immortal time (followed by 0 deaths) in 646 subjects accounts for $1,168.5$ person-years. The classification of this immortal time into the reference group decreases the rate of death in the reference group from 13.95 to 10.62 deaths $\cdot 100^{-1} \cdot \mathrm{yr}^{-1}$.

Table 3 shows that the mean time span between the first

Table 1.-Characteristics of cohort subjects according to regular therapy

\begin{tabular}{|c|c|c|c|c|}
\hline & \multicolumn{2}{|c|}{ Bronchodilator use (reference) } & \multicolumn{2}{|c|}{ ICS use } \\
\hline & $\begin{array}{l}\text { During entire } \\
\text { follow-up }\end{array}$ & $\begin{array}{l}\text { Followed by } \\
\text { ICS use }\end{array}$ & $\begin{array}{l}\text { First regular } \\
\text { therapy }\end{array}$ & $\begin{array}{c}\text { After bronchodilator } \\
\text { use }\end{array}$ \\
\hline Subjects $n$ & 1738 & 993 & 951 & 993 \\
\hline Subjects excluding $<6$ months follow-up ${ }^{\#} n$ & 1597 & 646 & 930 & 932 \\
\hline Age at cohort entry mean $\pm \mathrm{SD}$ & $74.7 \pm 9.4$ & $70.4 \pm 8.2$ & $71.1 \pm 8.6$ & $70.6 \pm 8.2$ \\
\hline Male sex $\%$ & 58.4 & 57.4 & 49.7 & 56.6 \\
\hline \multicolumn{5}{|l|}{ Use of COPD medications $\%$} \\
\hline SABA & 10.7 & 30.8 & 26.9 & 20.4 \\
\hline IB & 8.0 & 15.5 & 7.3 & 9.6 \\
\hline $\mathrm{SABA}+\mathrm{IB}$ & 0.9 & 7.0 & 0.5 & 1.3 \\
\hline Nebulised $\beta_{2}$-agonists & 8.0 & 12.5 & 3.4 & 6.1 \\
\hline Xanthines & 9.3 & 10.5 & 4.1 & 6.9 \\
\hline ICS & 2.1 & 0.0 & 11.2 & 7.6 \\
\hline Oral corticosteroids & 4.9 & 9.6 & 6.2 & 4.8 \\
\hline Antibiotics & 11.3 & 16.7 & 13.4 & 13.4 \\
\hline COPD hospitalisation $\%$ & 1.8 & 6.4 & 3.3 & 8.3 \\
\hline
\end{tabular}

ICS: inhaled corticosteroids; IB: ipratropium bromide; SABA: short-acting $\beta$-agonist; COPD: chronic obstructive pulmonary disease. ${ }^{\#}$ : to be consistent with criteria used in the previously published study [12]; ${ }^{\circ}$ : during 6 -month period prior to cohort entry.

Table 2. - Rates and crude rate ratios (RRs) of death for use of inhaled corticosteroids (ICS) from the Saskatchewan chronic obstructive pulmonary disease cohort, comparing the three approaches to data analysis

\begin{tabular}{|c|c|c|c|c|c|}
\hline Exposure to regular therapy & Subjects $n$ & Person-years & Deaths $n$ & Rate $^{+}$ & RR \\
\hline \multicolumn{6}{|c|}{ Hierarchical intention-to-treat analysis } \\
\hline ICS (all users) & 1862 & 4608.6 & 340 & 7.38 & 0.53 \\
\hline Bronchodilators $^{\#}$ (reference) & 1597 & 3726.9 & 520 & 13.95 & 1.00 \\
\hline \multicolumn{6}{|c|}{ Conventional intention-to-treat analysis } \\
\hline ICS & 930 & 2417.6 & 154 & 6.37 & 0.63 \\
\hline Bronchodilators (reference) & 2584 & 6457 & 649 & 10.05 & 1.00 \\
\hline \multicolumn{6}{|l|}{ According-to-treatment analysis } \\
\hline ICS & 1862 & 4608.6 & 340 & 7.38 & 0.69 \\
\hline Bronchodilators (reference) & 2243 & 4895.4 & 520 & 10.62 & 1.00 \\
\hline During entire follow-up & 1597 & 3726.9 & 520 & 13.95 & \\
\hline Prior to ICS & 646 & 1168.5 & 0 & 0.00 & \\
\hline
\end{tabular}

\#: only during follow-up; ${ }^{\uparrow}$ : immortal person-time in reference exposure prior to starting ICS exposure, these subjects are part of the 1862 subjects exposed to ICS; ${ }^{+}$: rate of all-cause death per 100 patients per year. 
Table 3.-Time span between first and third prescription dispensed within 6 months for either bronchodilators or inhaled corticosteroids (ICS), as used to define cohort entry

Bronchodilators ICS Difference (reference)

\begin{tabular}{llll}
\hline All subjects & & & \\
$\quad$ Subjects ${ }^{\#} \mathrm{n}$ & 2243 & 1862 & \\
$\quad$ Time mean days & 85.9 & 99.5 & 13.6 \\
Subjects with both exposures & 646 & 646 & \\
$\quad$ Subjects ${ }^{\#} \mathrm{n}$ & 86.2 & 98.4 & 12.2 \\
$\quad$ Time mean days & & \\
\hline \#: includes subjects who were regular bronchodilator users prior to \\
starting regular ICS use.
\end{tabular}

and third prescriptions is 85.9 days for bronchodilators and 99.5 days for ICS. When restricted to the subjects who belonged to both groups, the calculation gives similar results. These different time spans correspond to a difference in immortal time of $\sim 83.5$ person-yrs between the ICS and bronchodilator groups.

Table 4 shows the results of Cox's regression analysis for the three different approaches and both definitions of time zero. The crude RRs, obtained using the first prescription as defining time zero, are similar to the crude values estimated from the person-time approach in table 2. Generally, adjusted analyses increase the RR towards unity, as do the analyses based on the third prescription taken to define time zero. The adjusted RR for the according-to-treatment approach based on this latter definition is 0.94 (95\% confidence interval (CI) 0.81-1.09).

\section{Discussion}

In this study, a subtle but important bias was identified in the recently published observational study that suggested that regular ICS use with or without a long-acting bronchodilator is effective at reducing all-cause mortality in COPD patients. The study design, which bases its analysis on a hierarchical intention-to-treat approach to define the exposure groups, is biased from unaccounted immortal time. Using a comparable cohort of COPD patients identified from another database, from which the biased analysis was accurately reproduced, the current authors showed that the bias is sufficiently important

Table 4.-Crude and adjusted rate ratios (RRs) of death for inhaled corticosteroid use relative to bronchodilator use, from the Saskatchewan chronic obstructive pulmonary disease (COPD) cohort, comparing three approaches to data analysis and two cohort entry definitions

Crude
RR $\frac{\text { Adjusted }^{\#}}{\text { RR } 95 \% \text { CI }}$

Using first prescription as cohort entry Hierarchical intention-to-treat analysis Conventional intention-to-treat analysis According-to-treatment analysis

Using third prescription as cohort entry Hierarchical intent-to-treat analysis Conventional intention-to-treat analysis According-to-treatment analysis $\begin{array}{lll}0.52 & 0.66 & 0.57-0.76\end{array}$ $\begin{array}{lll}0.63 & 0.75 & 0.62-0.90\end{array}$ $\begin{array}{lll}0.67 & 0.85 & 0.73-0.98\end{array}$

$\begin{array}{lll}0.53 & 0.71 & 0.60-0.83\end{array}$

$\begin{array}{lll}0.57 & 0.72 & 0.58-0.88\end{array}$

$\begin{array}{llll}0.70 & 0.94 & 0.81-1.09\end{array}$
CI: confidence interval. \#: adjusted for age at cohort entry and sex, as well as the occurrence of COPD hospitalisations and the use of other COPD medications dispensed during the 6 -month period prior to cohort entry. that it rendered the significant effect nonsignificant. With the biased approach, it was found that the adjusted estimate of the $\mathrm{RR}$ of death for regular ICS use relative to regular bronchodilator use was 0.66 (95\% CI 0.57-0.76). After accounting for the two sources of immortal time bias introduced by this approach, the RR became 0.94 (95\% CI 0.81-1.09).

The bias results from overlooking two sources of immortal time. The bias is first introduced in the hierarchical definition of exposure. From a cohort of all newly diagnosed COPD patients, the hierarchy of exposure first assessed subjects from the date of diagnosis to the end of follow-up to identify the exposed, namely those who received three or more prescriptions of fluticasone proportionate or salmeterol during a 6-month period. The unexposed reference group is then defined by the subjects who did not receive any fluticasone proportionate or salmeterol during follow-up, but received three or more prescriptions of a short-acting bronchodilator during a 6-month period. Consequently, a large number of exposed subjects could have used short-acting bronchodilators prior to their start of fluticasone proportionate or salmeterol, as confirmed in table 1 [12]. This is plausible because of the steppedcare approach recommended in treatment guidelines for this disease, i.e. treatment should start with bronchodilators and corticosteroids should be added when the disease worsens [16]. Thus, several subjects from the exposed group were in fact unexposed before switching to this exposure status. More importantly, however, this pre-exposure time during which subjects were unexposed is an immortal period. These subjects, in switching from unexposed status to exposed status, will necessarily do so alive. Had they died before switching, they would, by definition, have belonged to the unexposed group. Thus, the bias occurs because valid unexposed persontime of follow-up with no deaths is not accounted for in the reference rate of death. This results in an artificial increase in the rate of death of the reference group, leading to a spurious appearance of effectiveness. The magnitude of the bias will then depend on the actual pattern and duration of steppingup therapy from short-acting bronchodilators to the addition of ICS and/or long-acting $\beta$-agonists. In the present study, analysis of the COPD cohort indicates that accounting for this first source of immortal time changes the rate ratio from 0.66 to 0.85 .

The previously published study focussed on fluticasone and salmeterol, suggesting that the combination is more effective than either drug, which are, in turn, more effective than shortacting bronchodilators alone. In this context, the hierarchical approach will accentuate the bias, as subjects receiving the combination could have first been exposed to short-acting bronchodilators, the reference, then to the long-acting bronchodilator or the ICS, before eventually being dispensed the latter two together during the same 6-month period. All immortal time spent in the reference exposure, as well as in the individual long-acting bronchodilator and ICS exposures, are not accounted for in the analysis. The present analysis suggests that the bias from unaccounted immortal time bias resulting from the hierarchical approach will lead to a hierarchy of effects.

The second source of immortal time arises from selecting time zero as the time of the first of the three prescriptions that define exposure. The time from the first to the third prescription is also immortal, as the subjects must have three prescriptions to satisfy the definition of regular use and are not included in the study if they die after the first or second. To avoid this bias, time zero should be defined as the date of the third of the three prescriptions, as has been done in previous similar studies of asthma and COPD [14, 17]. The analysis of the present COPD cohort indicates that accounting for this second source of immortal time changes the biased RR from 0.66 to 0.71 and the RR corrected for the first 
source of immortal time from 0.85 to 0.94 . In essence, the two sources of immortal time turn out to have an important impact on the RR of death for ICS use, changing it from a highly significant $0.66(95 \%$ CI $0.57-0.76)$ to a nonsignificant 0.94 (95\% CI 0.81-1.09).

The observational study presented here is also subject to the usual biases. ICS are expected to be prescribed to patients with more severe disease, who are at higher risk of death. Thus, the finding of a RR of 0.94 for ICS, even after adjustment for several confounding factors, may in fact suggest a modest benefit that must be investigated in large randomised trials. The diagnosis of COPD, from administrative databases, was only based on age and bronchodilator use. In the absence of information on smoking, symptoms and spirometry measures, subjects in the cohorts may have been misclassified as COPD. The unintended, yet possible, inclusion of asthma patients as COPD by these administrative criteria could lead to an apparent but fallacious beneficial effect of ICS in COPD. In addition, exposure was crudely defined to be consistent with the previously published cohort study [12]. Once a subject was allocated to an exposure group, they remained there and were considered exposed to that drug category until the end of follow-up, irrespective of whether they stopped or continued for the entire 3-yr follow-up. This assumption will produce exposure misclassification that tends to attenuate the RR towards unity.

As chronic obstructive pulmonary disease is one of the major causes of death, and as both mortality and morbidity due to chronic obstructive pulmonary disease have increased over the last two decades, the clinical implications of this observational study are important [18, 19]. While observational studies are a valuable addition to randomised controlled trials to demonstrate treatment effectiveness, they must be properly conducted to minimise bias [20]. Care must be taken in evaluating cohort studies with a hierarchical approach to exposure definition, as they can be subject to biases from unaccounted immortal time. This bias can give the false appearance of an important drug benefit, as can be seen with the apparent effectiveness of combination therapy with fluticasone and salmeterol to reduce mortality in chronic obstructive pulmonary disease. Randomised controlled trials are essential to verify whether these drugs, alone or in combination, are beneficial in chronic obstructive pulmonary disease. The important bias identified in this study, implies that the effectiveness of inhaled corticosteroids in chronic obstructive pulmonary disease remains debatable [21, 22].

Acknowledgements. The author would like to thank P. Ernst for helpful discussions and comments.

\section{References}

1. van Schayck CP, van Grunsven PM, Dekhuijzen PNR. Do patients with COPD benefit from treatment with inhaled cortocisteroids? Eur Respir J 1996; 9: 1969-1972.

2. Paggiaro PL, Dahle R, Bakran I, Frith L, Hollingworth K, Efthimiou J. Multicentre randomised placebo-controlled trial of inhaled fluticasone propionate in patients with chronic obstructive pulmonary disease. International COPD Study Group. Lancet 1998; 351: 773-780.

3. Bourbeau J, Rouleau MY, Boucher S. Randomised controlled trial of inhaled corticosteroids in patients with chronic obstructive pulmonary disease. Thorax 1998; 53: 477-482.

4. Vestbo J, Sorensen T, Lange P, Brix A, Torre P, Viskum K.
Long-term effect of inhaled budesonide in mild and moderate chronic obstructive pulmonary disease: a randomised controlled trial. Lancet 1999; 353: 1819-1823.

5. Pauwels RA, Lofdahl CG, Laitinen LA, et al. Long-term treatment with inhaled budesonide in persons with mild chronic obstructive pulmonary disease who continue smoking. European Respiratory Society Study on Chronic Obstructive Pulmonary Disease. N Engl J Med 1999; 340: 1948-1953.

6. The lung health study research group. Effect of inhaled triamcinolone on the decline in pulmonary function in chronic obstructive pulmonary disease. $N$ Engl $\mathrm{J} \mathrm{Med}$ 2000; 343: 1902-1909.

7. Burge PS, Calverley PM, Jones PW, Spencer S, Anderson JA, Maslen TK. Randomised, double blind, placebo controlled study of fluticasone propionate in patients with moderate to severe chronic obstructive pulmonary disease: the ISOLDE trial. BMJ 2000; 320: 1297-1303.

8. Sin DD, Tu JV. Inhaled corticosteroids and the risk of mortality and readmission in elderly patients with chronic obstructive pulmonary disease. Am J Respir Crit Care Med 2001; 164: 580-584.

9. Sin DD, Man SF. Inhaled corticosteroids and survival in chronic obstructive pulmonary disease: does the dose matter? Eur Respir J 2003; 21: 260-266.

10. Soriano JB, Kiri VA, Pride NB, Vestbo J. Inhaled corticosteroids with/without long-acting $\beta$-agonists reduce the risk of rehospitalization and death in COPD patients. $\mathrm{Am}$ J Respir Med 2003; 2: 67-74.

11. Suissa S. Effectiveness of inhaled corticosteroids in COPD: immortal time bias in observational studies. Am J Respir Crit Care Med 2003; 168: 49-53.

12. Soriano JB, Vestbo J, Pride NB, Kiri V, Maden C, Maier WC. Survival in COPD patients after regular use of fluticasone propionate and salmeterol in general practice. Eur Respir J 2002; 20: 819-825.

13. Downey W, Beck P, McNutt M, Stang M, Osei W, Nichol J. Health databases in Saskatchewan. In: Strom BL, ed. Pharmacoepidemiology. New-York, John Wiley \& Sons, Ltd, 2000; pp. 325-346.

14. Suissa S, Assimes T, Ernst P. Inhaled short acting beta agonist use in COPD and the risk of acute myocardial infarction. Thorax 2003; 58: 43-46.

15. Bourbeau J, Ernst P, Cockcoft D, Suissa S. Inhaled corticosteroids and hospitalisation due to exacerbation of COPD. Eur Respir J 2003; 22: 286-289.

16. Pauwels RA, Buist AS, Calverley PM, Jenkins CR, Hurd SS. Global strategy for the diagnosis, management, and prevention of chronic obstructive pulmonary disease. NHLBI/ WHO Global Initiative for Chronic Obstructive Lung Disease (GOLD) Workshop summary. Am J Respir Crit Care Med 2001; 163: 1256-1276.

17. Spitzer WO, Suissa S, Ernst $\mathrm{P}$, et al. The use of $\beta$-agonists and the risk of death and near death from asthma. $N$ Engl J Med 1992; 326: 501-506.

18. Hurd S. The impact of COPD on lung health worldwide: epidemiology and incidence. Chest 2000; 117: Suppl. 2, 1S$4 \mathrm{~S}$.

19. Anonymous. National Hospital Discharge Survey: 1980-1998. Hyattsville, Maryland, National Center for Health Statistics, 2000.

20. Samet JM. Measuring the effectiveness of inhaled corticosteroids for COPD is not easy! Am J Respir Crit Care Med 2003; 168: 1-2.

21. Calverley PM. Inhaled corticosteroids are beneficial in chronic obstructive pulmonary disease. Am J Respir Crit Care Med 2000; 161: 341-342.

22. Barnes PJ. Inhaled corticosteroids are not beneficial in chronic obstructive pulmonary disease. Am J Respir Crit Care Med 2000; 161: 342-344. 